\title{
Improved Finite-Difference Beam-Propagation Method Based on the Generalized Douglas Scheme and Its Application to Semivectorial Analysis
}

Junji Yamauchi, Member, IEEE, Jun Shibayama, Osamu Saito, Osamu Uchiyama, and Hisamatsu Nakano, Fellow, IEEE

\begin{abstract}
The generalized Douglas scheme for variable coefficients is applied to the propagating beam analysis. Once the alternating direction implicit method is used, the truncation error is reduced in the transverse directions compared with the conventional Crank-Nicholson scheme, maintaining a tridiagonal system of linear equations. Substantial improvement in the accuracy is achieved even in the TM mode propagation. As an example of the semivectorial analysis, the propagating field and the attenuation constant of a bent embedded waveguide with a trench section are calculated and discussed.
\end{abstract}

\section{INTRODUCTION}

$\mathbf{T}$ HE beam-propagation method (BPM) is widely used to design various optical circuits. In addition to the BPM based on the fast Fourier transform [1], the finite difference techniques are also applied to the propagating beam analysis [2]-[6]. It is well known that the implicit method such as the Crank-Nicholson $(\mathrm{CN})$ scheme allows the use of a larger propagation step length $\Delta z$ than the explicit method. The CN scheme has an advantage of unconditional stability, although the truncation error is $O(\Delta x)^{2}$ in the transverse direction.

To improve the accuracy, Yevick et al. [7] first introduced the Douglas scheme [8] into the propagating beam analysis. They applied the Douglas scheme to the Fresnel equation with the phase term being split. The truncation error is reduced to $O(\Delta x)^{4}$. It should be noted, however, that splitting the phase term results in the fact that the step length $\Delta z$ must be small. We can expect a larger step length when the phase term is not split [9].

Recently, we have formulated the modified finite-difference beam-propagation method (FD-BPM) based on the generalized Douglas (GD) scheme for variable coefficients [10], with the phase term being not split. The accuracy of our formulation is better than that of the previous one derived by Sun and Yip [11], maintaining the advantage that the result is not a sensitive function of $\Delta z$. The truncation error of $O(\Delta x)^{4}$ is ensured in the transverse direction.

The purpose of this paper is to discuss the application of the GD scheme to the propagating beam analysis in more detail. The GD scheme is applied to a semivectorial FD-BPM using the alternating direction implicit method (ADIM) [12]-[15]. Although the GD scheme holds approximately true in the

Manuscript received October 3, 1995; revised May 30, 1996.

The authors are with the College of Engineering, Hosei University, Tokyo 184, Japan.

Publisher Item Identifier S 0733-8724(96)07656-6. semivectorial FD-BPM, it still gives substantial improvement in the accuracy, as compared with the conventional $\mathrm{CN}$ scheme.

After demonstrating reduction of the mode-mismach loss in a step-index waveguide, we present numerical results of a three-dimensional embedded optical waveguide. Properties of quasi-TE and quasi-TM polarized modes are investigated by solving a semivectorial equation. The field distributions of a bent embedded waveguide are evaluated and the effects of a trench section [16]-[20] on the attenuation constant are discussed.

\section{SCALAR Formulation}

We first summarize the GD scheme in a two-dimensional problem. The Fresnel equation for the propagating beam problem is expressed as

$$
\sigma \frac{\partial E}{\partial z}=\frac{\partial^{2} E}{\partial x^{2}}+\nu E
$$

where $\sigma=2 j k n_{0}$ and $\nu=k^{2}\left[n^{2}(x, z)-n_{0}^{2}\right]$, in which $k$ is the free space wavenumber, $n(x, z)$ is the index profile of the waveguide, and $n_{0}$ is the reference index to be appropriately chosen.

The philosophy behind the Douglas scheme is to eliminate the truncation error term of the order $\Delta^{2}$ where $\Delta$ is the transverse sampling width. When the truncation error is included, by Taylor's series expansion the second derivative of function $E$ with respect to $x$ is expressed as

$$
\frac{\partial^{2} E}{\partial x^{2}}=\frac{\delta^{2} E}{\Delta x^{2}}-\frac{1}{12} \frac{\partial^{4} E}{\partial x^{4}} \Delta x^{2}+O(\Delta x)^{4} .
$$

In the conventional $\mathrm{CN}$ scheme, only the first term in the right-hand side of (1) is evaluated, so that the truncation error is $O(\Delta x)^{2}$.

To reduce the truncation error, we substitute (1) into (2), replacing $\partial^{4} E / \partial x^{4}$ with $\delta^{2}(\sigma \partial E / \partial z-\nu E) / \Delta x^{2}+O(\Delta x)^{2}$. Then, the following difference equation can be derived:

$$
\begin{aligned}
\frac{\left(\delta^{2} E\right)}{\Delta x^{2}}= & \frac{1}{12}\left(\sigma \frac{\partial E}{\partial z}\right)_{i+1}+\frac{5}{6}\left(\sigma \frac{\partial E}{\partial z}\right)_{i} \\
& +\frac{1}{12}\left(\sigma \frac{\partial E}{\partial z}\right)_{i-1}-\frac{1}{12}(\nu E)_{i+1}-\frac{5}{6}(\nu E)_{i} \\
& -\frac{1}{12}(\nu E)_{i-1}+O(\Delta x)^{4}
\end{aligned}
$$


We now introduce the $z$ differencing and get

$$
\begin{aligned}
\frac{\sigma}{12} & \frac{E_{i+1}^{l+1}-E_{i+1}^{l}}{\Delta z}+\frac{5 \sigma}{6} \frac{E_{i}^{l+1}-E_{i}^{l}}{\Delta z}+\frac{\sigma}{12} \frac{E_{i-1}^{l+1}-E_{i-1}^{l}}{\Delta z} \\
= & \frac{\left(\delta^{2} E\right)_{i}^{l+1}+\left(\delta^{2} E\right)_{i}^{l}}{2(\Delta x)^{2}}+\frac{1}{24}\left[(\nu E)_{i+1}^{l+1}+(\nu E)_{i+1}^{l}\right] \\
& +\frac{5}{12}\left[(\nu E)_{i}^{l+1}+(\nu E)_{i}^{l}\right]+\frac{1}{24}\left[(\nu E)_{i-1}^{l+1}+(\nu E)_{i-1}^{l}\right] \\
& +O(\Delta z)^{2}+O(\Delta x)^{4} .
\end{aligned}
$$

As a result, we obtain the following high accuracy six-point scheme:

$$
\begin{aligned}
& \zeta_{i-1}^{-} E_{i-1}^{l+1}+\xi_{i}^{-} E_{i}^{l+1}+\zeta_{i+1}^{-} E_{i+1}^{l+1} \\
& \quad=\zeta_{i-1}^{+} E_{i-1}^{l}+\xi_{i}^{+} E_{i}^{l}+\zeta_{i+1}^{+} E_{i+1}^{l}
\end{aligned}
$$

where

$$
\begin{aligned}
\zeta_{i}^{ \pm} & =\frac{j k n_{0}}{6} \pm \frac{\Delta z}{2 \Delta x^{2}} \pm \frac{\nu_{i} \Delta z}{24} \\
\xi_{i}^{ \pm} & =\frac{5 j k n_{0}}{3} \mp \frac{\Delta z}{\Delta x^{2}} \pm \frac{5 \nu_{i} \Delta z}{12} .
\end{aligned}
$$

It should be noted that the term $\nu$ is a variable coefficient. Inclusion of the $\nu_{i \pm 1}$ terms is significant for the reduction in the truncation error, as have been discussed previously [10]. Equation (4) can be regarded as the Douglas scheme generalized for variable coefficients. The six-point scheme allows us to use an efficient computing algorithm such as the Thomas algorithm, so that the computational speed is almost identical to that in the conventional FD-BPM [3] based on the $\mathrm{CN}$ scheme.

The scalar Fresnel equation in three-dimensional (3-D) coordinates are as follows:

$$
\sigma \frac{\partial E}{\partial z}=\frac{\partial^{2} E}{\partial x^{2}}+\frac{\partial^{2} E}{\partial y^{2}}+\nu E
$$

where it is difficult to directly derive a high-accuracy scheme. As described in [21], the difficulty arises from the fact that $(1 / 12) \Delta^{4}\left(\partial^{4} E / \partial x^{4}+\partial^{4} E / \partial y^{4}\right)$ cannot be expressed in terms of the Laplacian of $E$ in a square net of points in the $x-y$ plane. To obtain a high-accuracy scheme, we have to adopt a hexagonal net of points. This results in a 14-point scheme.

It should be noted, however, that once the Peaceman-Rachford ADIM [8], [12] is used, a high accuracy scheme can be obtained. The difference equation corresponding to (6) becomes

$$
\begin{aligned}
\sigma \frac{E^{l+1}-E^{l}}{\Delta z}= & \frac{\delta_{x}^{2} E^{l+1}+\delta_{x}^{2} E^{l}}{2 \Delta x^{2}}+\frac{\delta_{y}^{2} E^{l+1}+\delta_{y}^{2} E^{l}}{2 \Delta y^{2}} \\
& +\nu \frac{E^{l+1}+E^{l}}{2} .
\end{aligned}
$$

We divide the propagation step into two steps of size $\Delta z / 2$

$$
\begin{aligned}
\sigma \frac{E^{l+1 / 2}-E^{l}}{\Delta z / 2}= & \frac{\delta_{x}^{2} E^{l+1 / 2}}{\Delta x^{2}}+\frac{\delta_{y}^{2} E^{l}}{\Delta y^{2}}+\nu \frac{E^{l+1 / 2}+E^{l}}{2} \\
\sigma \frac{E^{l+1}-E^{l+1 / 2}}{\Delta z / 2}= & \frac{\delta_{x}^{2} E^{l+1 / 2}}{\Delta x^{2}}+\frac{\delta_{y}^{2} E^{l+1}}{\Delta y^{2}} \\
& +\nu \frac{E^{l+1}+E^{l+1 / 2}}{2}
\end{aligned}
$$

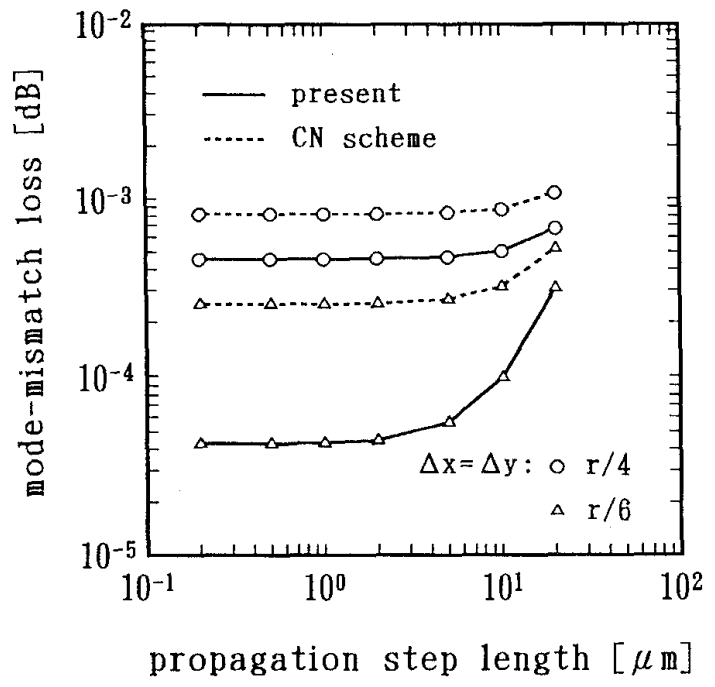

Fig. 1. Mode-mismatch loss for a step-index fiber as a function of propagation step length.

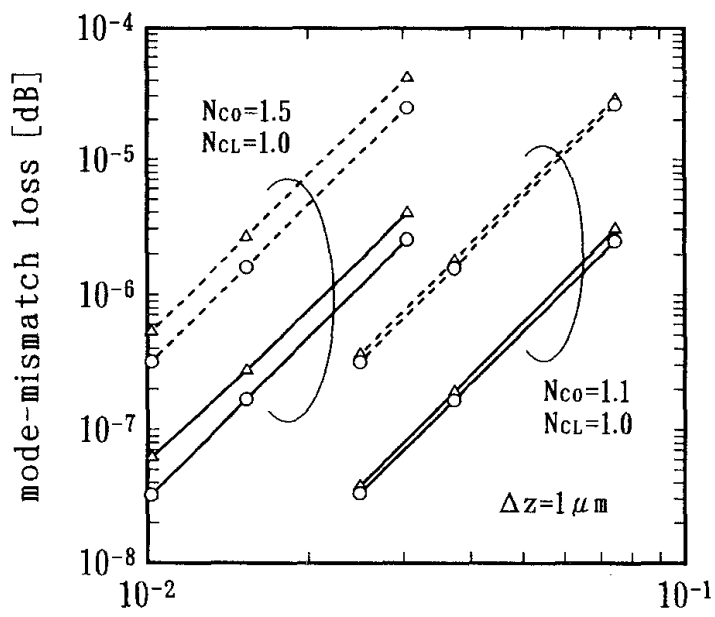

transverse sampling width $[\mu \mathrm{m}]$

Fig. 2. Mode-mismatch loss for a step-index slab waveguide as a function of transverse sampling width: __ present, ......... CN scheme, $\bigcirc \mathrm{TE}_{0}$ mode, $\triangle \mathrm{TM}_{0}$ mode.

Since the treatment regarding the two half-steps is the same, we only discuss the first half-step in the following.

Equation (8) can be separated into

$$
\begin{gathered}
\left(\sigma \frac{\partial E}{\partial z}\right)^{l}=2\left(\frac{\partial^{2} E}{\partial y^{2}}\right)^{l}+\nu E^{l} \\
\left(\sigma \frac{\partial E}{\partial z}\right)^{l+1 / 2}=2\left(\frac{\partial^{2} E}{\partial x^{2}}\right)^{l+1 / 2}+\nu E^{l+1 / 2} .
\end{gathered}
$$

This is confirmed by the fact that the integration of $\sigma(\partial E / \partial z)$ in the interval $[z, \Delta z / 2]$ using (10) and (11) results in (8). Since the forms of (10) and (11) are almost the same as (1), we can use the GD scheme even in a 3-D problem. The efficient 


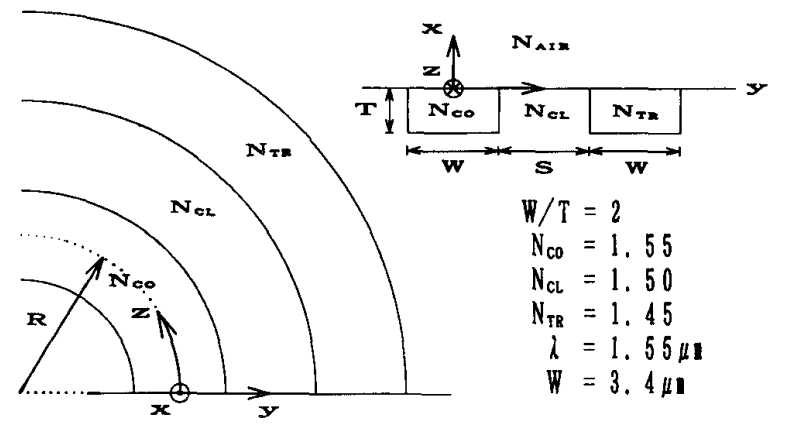

Fig. 3. Configuration of a bent embedded optical waveguide.

computing algorithm can again be used as in the case of a two-dimensional problem. As a result, the following difference equations split into two steps are derived:

$$
\begin{aligned}
& \zeta_{x i-1, j}^{-} E_{i-1, j}^{l+1 / 2}+\xi_{x i, j}^{-} E_{i, j}^{l+1 / 2}+\zeta_{x i+1, j}^{-} E_{i+1, j}^{l+1 / 2} \\
& \quad=\zeta_{y i, j-1}^{+} E_{i, j-1}^{l}+\xi_{y i, j}^{+} E_{i, j}^{l}+\zeta_{y i, j+1}^{+} E_{i, j+1}^{l} \\
& \zeta_{y i, j-1}^{-} E_{i, j-1}^{l+1}+\xi_{y i, j}^{-} E_{i, j}^{l+1}+\zeta_{y i, j+1}^{-} E_{i, j+1}^{l+1} \\
& \quad=\zeta_{x i-1, j}^{+} E_{i-1, j}^{l+1 / 2}+\xi_{x i, j}^{+} E_{i, j}^{l+1 / 2}+\zeta_{x i+1, j}^{+} E_{i+1, j}^{l+1 / 2}
\end{aligned}
$$

in which

$$
\begin{aligned}
\zeta_{\alpha i, j}^{ \pm} & =\frac{j k n_{0}}{6} \pm \frac{\Delta z}{2 \Delta \alpha^{2}} \pm \frac{\nu_{i, j} \Delta z}{48}, \\
\xi_{\alpha i, j}^{ \pm} & =\frac{5 j k n_{0}}{3} \mp \frac{\Delta z}{\Delta \alpha^{2}} \pm \frac{5 \nu_{i, j} \Delta z}{24}
\end{aligned}
$$

where $\alpha=x$ or $y$.

At the edge of the computational window the transparent boundary condition (TBC) [22] is imposed. The TBC may fail for radiation fields with an appreciable wave vector spread. However, as will be shown later, the TBC works well in the analysis of a bent waveguide. This is due to the fact that the radiation field is dominated by a single transverse wave vector component.

To demonstrate the effectiveness of the GD scheme, we first investigate the propagation error of the fundamental mode $\mathrm{LP}_{01}$ in a step-index optical fiber. We evaluate the following mode-mismatch loss $L_{M}$

$$
L_{M}=-10 \log \left(\left|\int E_{0} E^{*} d x d y\right|^{2} /\left[\int\left|E_{0}\right|^{2} d x d y\right]^{2}\right)[\mathrm{dB}]
$$

where $E$ is the propagating field and $E_{0}$ is the incident field of the fundamental mode. The mode-mismatch loss is known to be a sensitive indicator of the accuracy of a beam-propagation method [3].

The fiber has a core radius of $r=5 \mu \mathrm{m}$, and the refractive indices of $N_{\mathrm{CO}}=1.504$ and $N_{\mathrm{CL}}=1.500$. A stepped approximation is used for the circular core [18]. The computational window dimensions are given by $L_{x} \times L_{y}=125 \times 125 \mu \mathrm{m}^{2}$ and the wavelength is $\lambda=1.55 \mu \mathrm{m}$. In the analysis, the reference index is chosen to be that in the cladding.
Fig. 1 shows the mode-mismatch loss evaluated at $z=$ $500 \mu \mathrm{m}$ as a function of propagation step length $\Delta z$. For comparison, the data obtained from the conventional ADIM based on the $\mathrm{CN}$ scheme are also presented. It is found that the accuracy of the GD scheme is better than that of the CN scheme. The numerical result is not a sensitive function of $\Delta z$.

\section{SEMIVECTORIAL Formulation}

Consideration is next given to the semivectorial analysis, in which we solve the scalar wave equation but accounts for the discontinuity in fields caused by the index variation. The semivectorial Fresnel equation is expressed as

$$
\sigma \frac{\partial E}{\partial z}=\frac{\partial}{\partial x}\left(\frac{1}{n^{2}}\left\{\frac{\partial}{\partial x}\left(n^{2} E\right)\right\}\right)+\frac{\partial^{2} E}{\partial y^{2}}+\nu E .
$$

Equation (15) can also be solved by the ADIM. Equations corresponding to (10) and (11) are, respectively

$$
\begin{aligned}
\left(\sigma \frac{\partial E}{\partial z}\right)^{l} & =2\left(\frac{\partial^{2} E}{\partial y^{2}}\right)^{l}+\nu E^{l} \\
\left(\sigma \frac{\partial E}{\partial z}\right)^{l+1 / 2} & =2 \frac{\partial}{\partial x}\left(\frac{1}{n^{2}}\left\{\frac{\partial}{\partial x}\left(n^{2} E\right)\right\}\right)^{l+1 / 2}+\nu E^{l+1 / 2} .
\end{aligned}
$$

The second derivative with respect to $y$ can be treated as in the case of (3). Unfortunately, the Taylor's series expansion regarding the second derivative with respect to $x$ is not simple because of the existence of the index term. Therefore, by analogy with (2) we assume the following relation based on the Stern's expression [23]

$$
\begin{aligned}
\frac{\partial}{\partial x}\left(\frac{1}{n^{2}}\left\{\frac{\partial}{\partial x}\left(n^{2} E\right)\right\}\right)= & \frac{a_{i} E_{i-1}-2 b_{i} E_{i}+c_{i} E_{i+1}}{\Delta x^{2}} \\
& -\frac{1}{12} \delta^{2}\left(\sigma \frac{\partial E}{\partial z}-\nu E\right)
\end{aligned}
$$

in which

$$
\begin{aligned}
a_{i} & =\frac{2 n_{i-1}^{2}}{n_{i-1}^{2}+n_{i}^{2}} \\
b_{i} & =n_{i}^{2}\left(\frac{1}{n_{i-1}^{2}+n_{i}^{2}}+\frac{1}{n_{i}^{2}+n_{i+1}^{2}}\right) \\
c_{i} & =\frac{2 n_{i+1}^{2}}{n_{i+1}^{2}+n_{i}^{2}}
\end{aligned}
$$

Although (18) dose not ensure the truncation error of $O(\Delta x)^{4}$, it leads to substantial improvement in accuracy as will be found later.

After some manipulations, we obtain the following difference equations:

$$
\begin{aligned}
& \zeta_{x i-1, j}^{-} E_{i-1, j}^{l+1 / 2}+\xi_{x i, j}^{-} E_{i, j}^{l+1 / 2}+\eta_{x i+1, j}^{-} E_{i+1, j}^{l+1 / 2} \\
& \quad=\zeta_{y i, j-1}^{+} E_{i, j-1}^{l}+\xi_{y i, j}^{+} E_{i, j}^{l}+\zeta_{y i, j+1}^{+} E_{i, j+1}^{l} \\
& \zeta_{y i, j-1}^{-} E_{i, j-1}^{l+1}+\xi_{y i, j} E_{i, j}^{l+1}+\zeta_{y i, j+1}^{-} E_{i, j+1}^{l+1} \\
& \quad=\zeta_{x i-1, j}^{+} E_{i-1, j}^{l+1 / 2}+\xi_{x i, j}^{+} E_{i, j}^{l+1 / 2}+\eta_{x i+1, j}^{+} E_{i+1, j}^{l+1 / 2}
\end{aligned}
$$




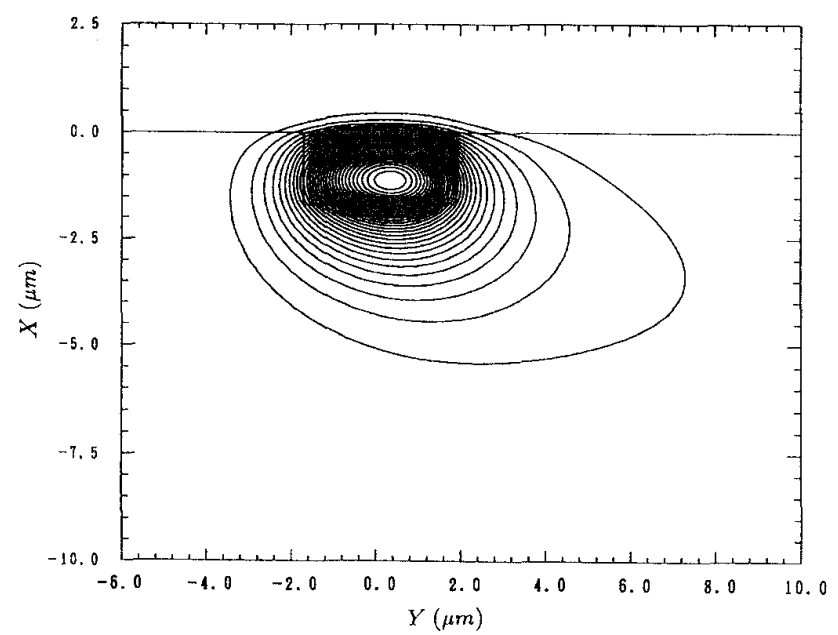

(a)

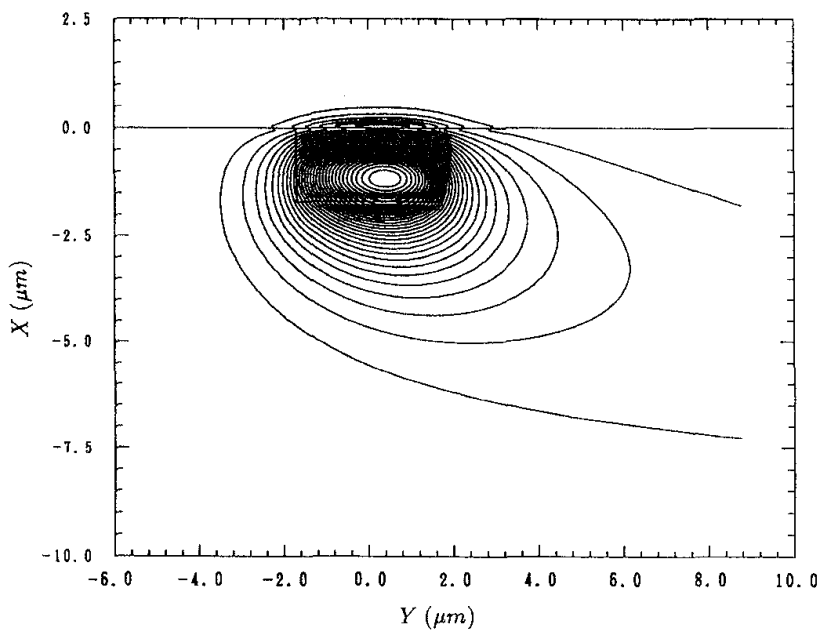

(c)

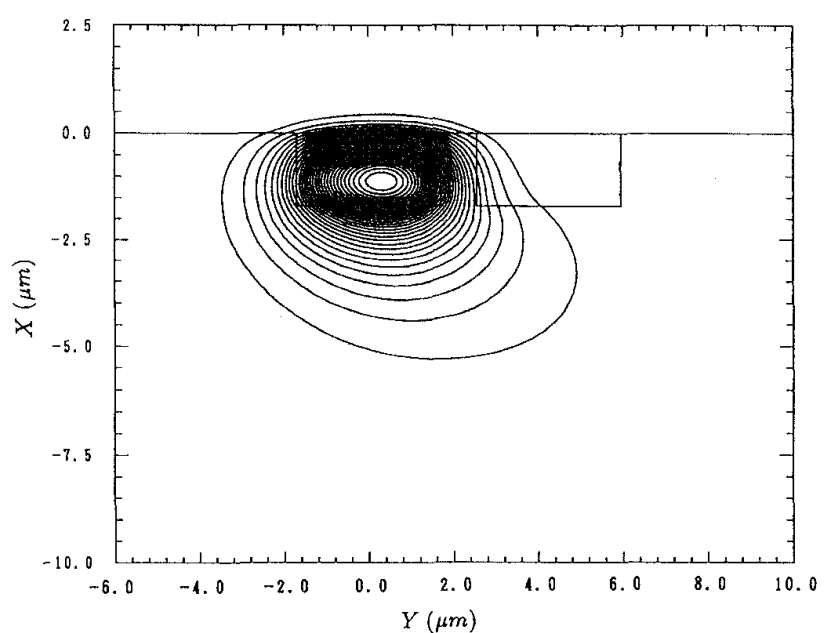

(b)

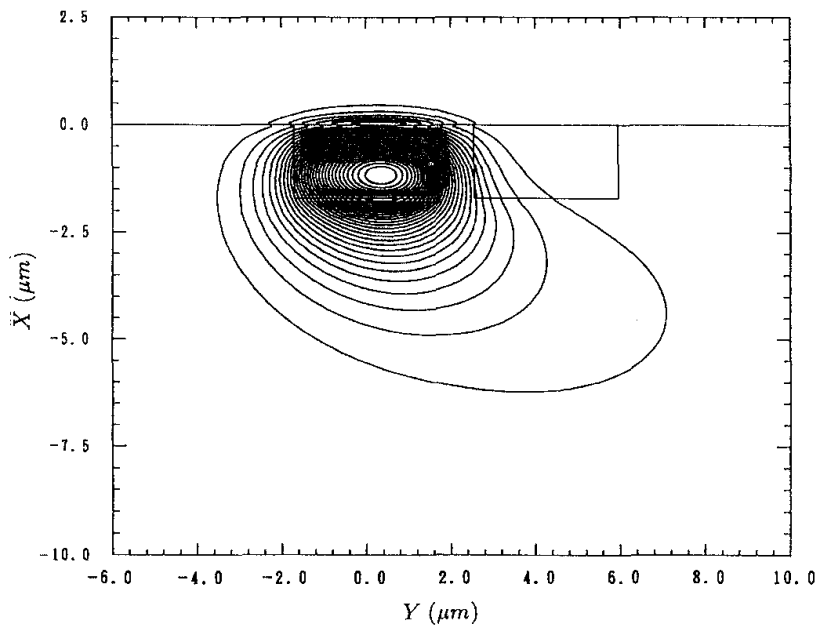

(d)

Fig. 4. Field distributions of a bent embedded optical waveguide. (a) Quasi-TE mode without trench, (b) quasi-TE mode with trench, (c) quasi-TM mode without trench, and (d) quasi-TM mode with trench.

in which

$$
\begin{aligned}
\zeta_{x i, j}^{ \pm} & =\frac{j k n_{0}}{6} \pm \frac{\Delta z}{2 \Delta x^{2}} a_{i, j} \pm \frac{\nu_{i, j} \Delta z}{48} \\
\xi_{x i, j}^{ \pm} & =\frac{5 j k n_{0}}{3} \mp \frac{\Delta z}{\Delta x^{2}} b_{i, j} \pm \frac{5 \nu_{i, j} \Delta z}{24} \\
\eta_{x i, j}^{ \pm} & =\frac{j k n_{0}}{6} \pm \frac{\Delta z}{2 \Delta x^{2}} c_{i, j} \pm \frac{\nu_{i, j} \Delta z}{48} \\
\zeta_{y i, j}^{ \pm} & =\frac{j k n_{0}}{6} \pm \frac{\Delta z}{2 \Delta y^{2}} \pm \frac{\nu_{i, j} \Delta z}{48} \\
\xi_{y i, j}^{ \pm} & =\frac{5 j k n_{0}}{3} \mp \frac{\Delta z}{\Delta y^{2}} \pm \frac{5 \nu_{i, j} \Delta z}{24}
\end{aligned}
$$

To verify the improvement in the numerical accuracy of the semivectorial analysis, we treat two step-index slab waveguides with a different core index $N_{\mathrm{CO}}$. The propagation of $\mathrm{TM}_{0}$ mode is studied together with that of $\mathrm{TE}_{0}$ mode. The core width is designated as $2 D$. The normalized frequency is taken to be $V=1.5$. The cladding index $N_{\mathrm{CL}}$ is fixed to be
1.0. The computational window dimensions are $6.10 \mu \mathrm{m}$ and $14.88 \mu \mathrm{m}$ for $N_{\mathrm{CO}}=1.5$ and 1.1 , respectively. This means that the number of transverse sampling points is in a range of 200 to 600 depending on $\Delta x$ under the condition of the fixed window dimension. The wavelength is $\lambda=1 \mu \mathrm{m}$. The loss evaluation is carried out at a propagation distance of $500 \mu \mathrm{m}$.

Fig. 2 shows the mode-mismatch loss as a function of transverse sampling width. For comparison, the results obtained by the $\mathrm{CN}$ scheme are also shown. The mode-mismatch loss observed for the GD scheme is found to be much smaller than that for the $\mathrm{CN}$ scheme. It is worth mentioning that the GD scheme is effective even in the TM mode.

As an application of the GD scheme to a three-dimensional waveguide, we analyze a bent embedded waveguide shown in Fig. 3. The refractive indexes are $N_{\mathrm{CO}}=1.55$ and $N_{\mathrm{CL}}=$ 1.50. The wavelength of $\lambda=1.55 \mu \mathrm{m}$ is used. The aspect ratio of $W / T$ is chosen to be 2 , and the width $W$ is taken to be $3.4 \mu \mathrm{m}$, so that only the fundamental mode propagates. The 


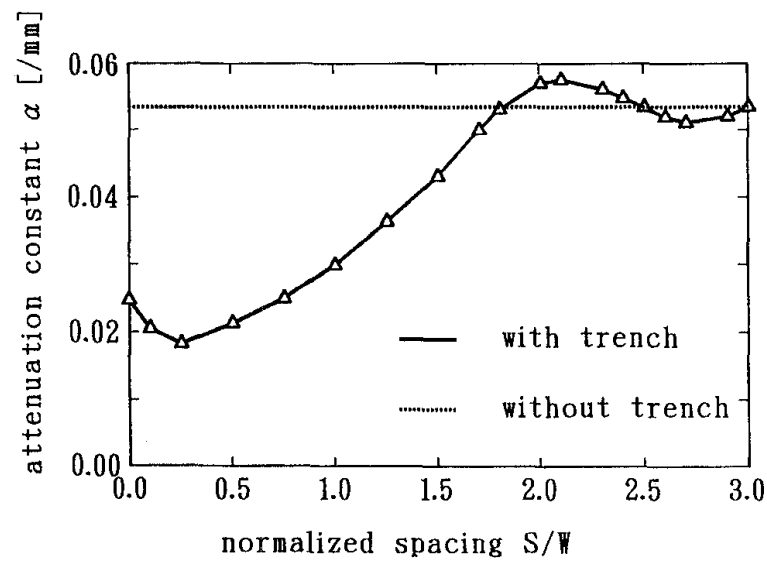

(a)

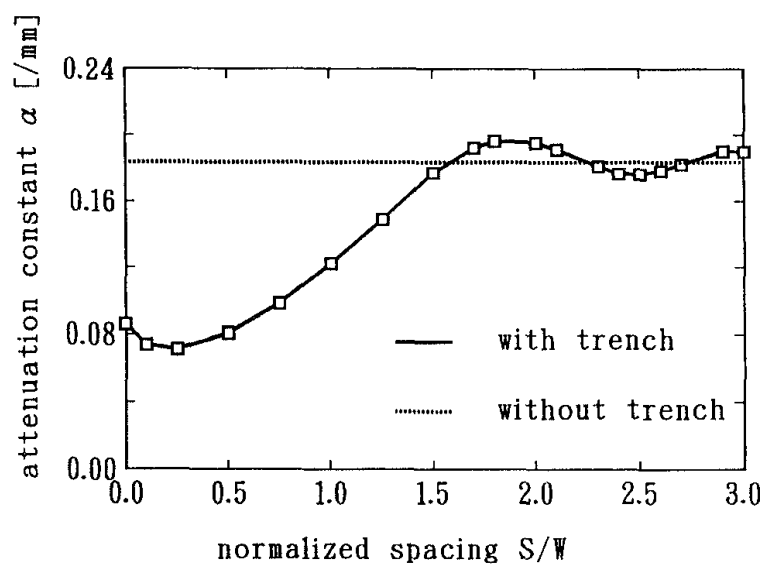

(b)

Fig. 5. Attenuation constant as a function of spacing between core and trench. (a) Quasi-TE mode and (b) quasi-TM mode.

bending radius is $R=500 \mu \mathrm{m}$. The computation parameters are as follows; $\Delta x=\Delta y / 2=0.085 \mu \mathrm{m}, \Delta z=0.25 \mu \mathrm{m}$, the numbers of transverse sampling points are $N_{x}=120$ and $N_{y}=160$. The fundamental-mode field is excited at $z=0$. The index profile of the waveguide bend is transformed into that of an equivalent straight waveguide [24].

Fig. 4(a) and (c) shows the steady-state field distribution observed at a propagation distance of $1200 \mu \mathrm{m}$. It is seen that the field deforms toward the outer side of the bend. The deformation of the field for the quasi-TM mode is found to be larger than that for the quasi-TE mode. This results in a larger attenuation constant in the quasi-TM mode as will be shown in Fig. 5.

To suppress the field deformation, we place the trench section at the outer side of the bend, as shown in Fig. 3. The width of the trench is the same as that of the core. The refractive index in the trench is $N_{\mathrm{TR}}=1.45$, and the spacing between the core and the trench is $S=W / 4$. Figures 4(b) and (d) show how the trench enhances the field evanescence.

Effects of the trench location on the attenuation constant $\alpha$ are shown in Fig. 5. The attenuation constant is evaluated using

$$
\alpha=-\frac{1}{2 \Delta z} \ln \left(\frac{P(z+\Delta z)}{P(z)}\right) .
$$

The ADIM with the phase term being not split does not conserve power. Therefore, we should check that $\alpha$ evaluated for the straight waveguide is sufficiently smaller than that caused by the bend. Preliminary calculation shows that $\alpha$ for the straight waveguide is less than $0.00004 / \mathrm{mm}$ for both modes, and is negligible in the following discussion.

Fig. 5 shows that the attenuation constant is small when the trench is made near the core. The appropriate location of the trench is close to the so-called radiation point, where the field becomes radiative. In this case, the appropriate location is around $S=W / 4$ regardless of the polarization. It is also seen that $\alpha$ exhibits oscillatory behavior as a function of spacing $S$, as in the case of a two-dimensional step-index slab waveguide [19]. As discussed in detail in [20], this behavior can be explained by the fact that the sandwiched region between the core and the trench turns to a quasiwaveguiding region whose effective index changes as a function of spacing $S$. As expected, $\alpha$ converges to a value without the trench as the spacing $S$ is further increased.

\section{CONCLUSION}

The improved FD-BPM based on the generalized Douglas scheme for variable coefficients, in which the phase term is not split, has been discussed. The use of the Peaceman-Rachford ADIM achieves the reduced truncation error in the transverse directions, maintaining a tridiagonal system of linear equations. The computational time is almost identical to that in the conventional $\mathrm{CN}$ scheme. The mode-mismatch loss calculation shows that the present scheme is effective even in the analysis of TM mode propagation. As an example of the semivectorial analysis, the field deformation and the attenuation constant of a bent embedded waveguide are evaluated. Calculation shows how the trench enhances the field evanescence with subsequent reduction in the attenuation constant.

\section{REFERENCES}

[1] M. D. Feit and J. A. Fleck, $\mathrm{J}_{\mathrm{r} .,}$ "Light propagation in graded index fibers," Appl. Opt., vol. 17, no. 24, pp. 3990-3998, 1978.

[2] D. Yevick and B. Hermansson, "Split-step finite difference analysis of rib waveguides," Electron. Lett., vol. 25, no. 7, pp. 461-462, 1989.

[3] Y. Chung and N. Dagli, "An assessment of finite difference beam propagation method," IEEE J. Quantum Electron., vol. 26, pp. 1335--1339, Aug. 1990.

[4] R. Accornero, M. Artiglia, G. Coppa, P. Di Vita, G. Lapenta, M. Potenza, and P. Ravetto, "Finite difference methods for the analysis of integrated optical waveguides," Electron. Lett., vol. 26, no. 23, pp. 1959-1960, 1990.

[5] R. Scarmozzino and R. M. Osgood, Jr., "Comparison of finite-difference and Fourier-transform solutions of the parabolic wave equation with emphasis on integrated-optics applications," J. Opt. Soc. Amer., A, vol. 8 , no. 5 , pp. $724-731,1991$.

[6] Y. Chung and N. Dagli, "Analysis of $z$-invariant and $z$-variant semiconductor rib waveguides by explicit finite difference beam propagation method with nonuniform mesh configuration," IEEE J. Quantum Electron., vol. 27, pp. 2296-2305, Oct. 1991.

[7] D. Yevick, B. Hermansson, and M. Glasner, "Fresnel and wide-angle equation analyses of microlenses," IEEE Photon. Technol. Lett., vol. 2, pp. 412-414, June 1990. 
[8] A. R. Mitchell and D. F. Griffiths, The Finite Difference Method in Partial Differential Equations. New York: Wiley, 1987, ch. 2.

[9] D. Yevick and B. Hermansson, "Efficient beam propagation techniques," IEEE J. Quantum Electron., vol. 26, pp. 109-112, Jan. 1990.

[10] J. Yamauchi, J. Shibayama, and H. Nakano, "Modified finite-difference beam propagation method based on the generalized Douglas scheme for variable coefficients," IEEE Photon. Technol. Lett., vol. 7, pp. 661-663, June 1995.

[11] L. Sun and G. L. Yip, "Modified finite-difference beam-propagation method based on the Douglas scheme," Opt. Lett., vol. 18, no. 15, pp. $1229-1231,1993$

[12] W. H. Press, B. P. Flannery, S. A. Teukolsky, and W. T. Vetterling, Numerical Recipes: The Art of Scientific Computing. New York: Cambridge University Press, 1986, ch. 17.

[13] J. Yamauchi, T. Ando, and H. Nakano, "Beam-propagation anlaysis of optical fibers by alternating direction implicit method," Electron. Lett., vol. 27 , no. 18 , pp. 1663-1665, 1991.

[14] P. L. Liu and B. J. Li, "Semivectorial beam-propagation method for analyzing polarized modes of rib waveguides," IEEE J. Quantum Electron., vol. 28, pp. 778-782, Apr. 1992.

[15] "The semivectorial beam propagation method" IEEE J. Quantum Electron., vol. 29, pp. 1205-1211, Apr. 1993.

[16] S. Kawakami, M. Miyagi, and S. Nishida, "Bending losses of dielectric slab optical waveguides with double or multiple claddings: Theory," Appl. Opt., vol. 14, no. 11, pp. 2588-2597, 1975

[17] E. G. Neumann, "Low loss dielectric optical waveguide bends," Fiber and Integrated Optics, vol. 4, no. 2, pp. 203-211, 1982.

[18] J. Yamauchi, M. Ikegaya, and H. Nakano, "Analysis of bent step-index optical fibers by the beam propagation method," IEE Proc. $J$, vol. 139, no. 3, pp. 201-207, 1992.

[19] J. Yamauchi, M. Ikegaya, and H. Nakano, "Bend loss of step-index slab waveguides with a trench section," Microwave \& Opt. Technol. Lett., vol. 5 , no. 6 , pp. 251-254, 1992.

[20] J. Yamauchi, T. Ando, M. Ikegaya, and H. Nakano, "Effects of trench location on the attenuation constant in bent step-index optical waveguides," IEICE Trans. Electron,, vol. E77-C, no. 2, pp. 319-321, 1994.

[21] R. D. Richtmyer and K. W. Morton, Difference Methods for Initial-Value Problems. New York: Wiley, 1967, 2nd ed., ch. 8

[22] G. R. Hadley, "Transparent boundary condition for beam propagation," Opt. Lett., vol. 16, no. 9, pp. 624-626, 1991.

[23] M. S. Stern, "Semivectorial polarized finite difference method for optical waveguides with arbitrary index profiles," IEE Proc. J., vol. 135, no. 1, pp. 56-63, 1988

[24] J. Saijonmaa and D. Yevick, "Beam-propagation analysis of loss in bent optical waveguides and fibers," J. Opt. Soc. Amer., vol. 73, no. 12, pp. $1785-1791,1983$

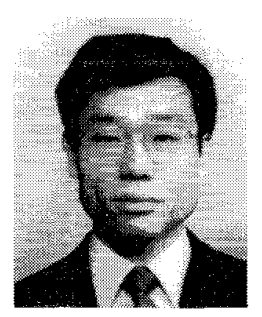

Junji Yamauchi (M'85) was born in Nagoya, Japan, on August 23, 1953. He received the B.E., M.E., and Dr.E. degrees from Hosei University, Tokyo, Japan, in 1976,1978 , and 1982, respectively.

From 1984 to 1988 , he served as a Lecture at the Electrical Engineering Department of Tokyo Metropolitan Technical College. Since 1988, he has been a member of the faculty of Hosei University, where he is now a Professor of Electronic Informatics. His research interests include optical waveguides and circularly polarized antennas.

Dr. Yamauchi is a member of the Optical Society of America and the Institute of Electronics, Information and Communication Engineers of Japan (IEICE).

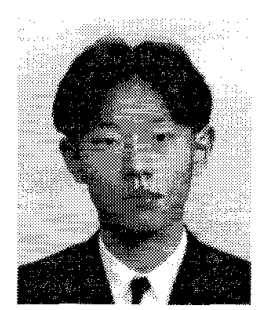

Jun Shibayama was born in Chiba, Japan, on July 1, 1969. He received the B.E. and M.E. degrees from Hosei University, Tokyo, Japan, in 1993 and 1995, respectively.

He joined Furukawa Electric Company, Ltd. Tokyo in 1995.

Mr. Shibayama is a member of the Institute of Electronics, Information and Communication Engincers of Japan (IEICE)

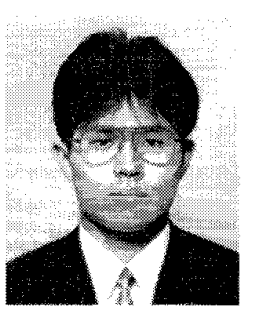

Osamu Saito was born in Saitama, Japan, on February 4,1970 . He received the B.E. and M.E. degrees from Hosei University, Tokyo, Japan, in 1993 and 1995, respectively.

He joined Chuo Electric Company, Tokyo in 1995.

Mr. Saito is a member of the Institute of Electronics, Information and Communication Engineers of Japan (IEICE).

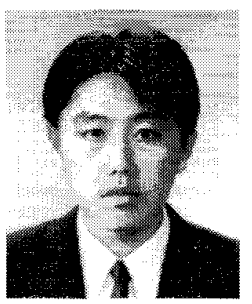

Osamu Uchiyama received the B.S. degree in electrical engineering from Hosei University, Tokyo, Japan, in 1995, where he is presently pursuing the Master's degree.

$\mathrm{He}$ is involved in research on numerical analysis of optical waveguides.

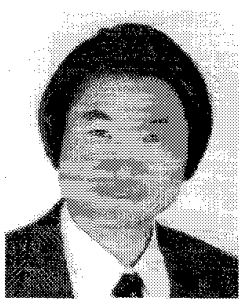

Hisamatsu Nakano (M'75-SM'87-F'92) was born in Ibaraki, Japan, on April 13, 1945. He received the B.E., M.E., and Dr.E. degrees in electrical engineering from Hosei University, Tokyo, Japan, in 1968,1970 , and 1974, respectively.

Since 1973 , he has been a member of the faculty of Hosei University, where he is now a Professor of Electronic Informatics. His research topics include numerical methods for microwave antennas and scattering problems. He has published more than 120 refereed papers on antenna and relevant problems. He is the author of the book entitled Helical and Spiral Antennas (New York: Research Studies Press, Wiley). In 1995, he published the chapter "Antenna Analysis Using Integral Equations," in the book entitled Analysis Methods of Electromagnetic Wave Problems (E. Yamashita, Editor, vol. 2). His recent interests have been in the development of a parabolic antenna using backfire helical feed for direct reception of broadcasting satellite TV programs (DBS). He has also been involved in the development of two types of small indoor flat DBS antennas using novel elements: curled and extremely low-profile helical elements. His other interests include microstrip antennas for grobal positioning systems. He was a Visiting Associate Professor at Syracuse University, Syracuse, NY (May to September 1991), where he worked on numerical analysis of electromagnetic coupling between wires and slots, a Visiting Professor at University of Manitoba, Canada (March to September 1986), researching numerical techniques for analysis of microstrip antennas, and a Visiting Professor at University of California, Los Angeles (September 1986 to March 1987), working on microstrip line antenna analysis.

Dr. Nakano was the recipient of an International Scientific Exchange Award from the Natural Sciences and Engineering Research Council of Canada. In 1987, he received the Best Paper Award of the IEE Fifth International Conference on Antennas and Propagation. In 1994, he received the H. A. Wheeler Applications Prize Paper Award. He is an Associate Editor of IEEE antennas and Propagation Magazine. 